\title{
ACTIVIDAD ACETILCOLINESTERASA (AChE) Y BUTIRILCOLINESTERASA (BUChE) EN POBLACIONES MEXICANAS: ESTUDIO PILOTO
}

\author{
Yael Yvette BERNAL-HERNÁNDEZ ${ }^{1 *}$, Daniela AGUILERA-MÁRQUEZ², Patricia GRAJEDA-COTA ${ }^{3}$, \\ Gladys Alejandra TOLEDO-IBARRA ${ }^{4}$, María Elena MORENO-GODÍNEZ ${ }^{5}$, \\ Javier Humberto PERERA-RÍOS ${ }^{6}$, Maytut Osdely URIÓSTEGUI-ACOSTA ${ }^{7}$, \\ Aurora Elizabeth ROJAS-GARCÍA ${ }^{1}$, Irma Martha MEDINA-DÍAZ ${ }^{1}$, \\ Briscia Socorro BARRÓN-VIVANCO ${ }^{1}$ y Cyndia Azucena GONZÁLEZ-ARIAS ${ }^{1}$
}

${ }^{1}$ Laboratorio de Contaminación y Toxicología Ambiental, Universidad Autónoma de Nayarit

${ }^{2}$ Centro de Investigación en Alimentación y Desarrollo, Unidad Guaymas

${ }^{3}$ Coordinación de Ciencia de los Alimentos, Centro de Investigación en Alimentación y Desarrollo, Unidad Hermosillo

${ }^{4}$ Laboratorio Nacional para la Investigación en Inocuidad Alimentaria (LANIIA), Unidad Nayarit, Centro Nayarita de Innovación y Transferencia de Tecnología, A.C., Tepic, Nayarit

${ }^{5}$ Laboratorio de Toxicología y Salud Ambiental, Unidad Académica de Ciencias Químico-Biológicas, Universidad Autónoma de Guerrero

${ }^{6}$ Laboratorio de Enfermedades Crónicas y Degenerativas (LECD), Universidad Autónoma de Yucatán

${ }^{7}$ Unidad Académica de Ciencias Químico Biológicas, Universidad Autónoma de Guerrero

*Autor para correspondencia: yael.yvette@gmail.com

(Recibido enero 2017; aceptado diciembre 2017)

Palabras clave: plaguicidas, colinesterasas, factores preanalíticos y analíticos

\section{RESUMEN}

La determinación de las actividades acetilcolinesterasa (AChE) y butirilcolinesterasa (BuChE) es ampliamente utilizada en estudios de monitoreo biológico para evaluar la exposición a compuestos anticolinesterásicos y sus efectos. No obstante, se ha observado una amplia variabilidad en los valores considerados como normales para estas actividades enzimáticas, lo que puede deberse a diversos factores. Uno de los que puede tener más influencia es la falta de parámetros preanalíticos y analíticos estandarizados. En este sentido, el objetivo de este trabajo fue conocer, con una metodología consensuada, los intervalos de las actividades $\mathrm{AChE}$ y BuChE en individuos no expuestos ocupacionalmente a compuestos anticolinesterásicos en diferentes estados de la república mexicana. Se observó que el $22 \%$ de los laboratorios participantes reportaron valores de actividad AChE dentro del rango considerado como normal. Respecto a la actividad BuChE, el 67 \% reportó valores dentro del rango para una población no expuesta a compuestos anticolinesterásicos. Los resultados revelaron una importante variabilidad en los valores de las actividades AChE y BuChE, que muestran la necesidad de capacitación continua entre los analistas y la homogeneización de las metodologías.

Key words: pesticides, cholinesterase activity, preanalytical and analytical factors 


\begin{abstract}
The analytical determination of acetylcholinesterase (AChE) and butyrylcholinesterase $(\mathrm{BuChE})$ activities is widely used in biological biomonitoring to evaluate exposure and detrimental effects caused by anticholinesterasic compounds. Nevertheless, a wide variability in values within the range considered as normal has been reported, which could be due to different factors. One of the most influencing factors on the variability of the data is the lack of standardized analytical and preanalytical parameters. The aim of this work was to know, under a consensual methodology, the intervals of $\mathrm{AChE}$ and $\mathrm{BuChE}$ activities in individuals non-occupationally exposed to anticholinesterase compounds from different states of the Mexican republic. From the participating laboratories, $22 \%$ reported values of $\mathrm{AChE}$ activity within the range considered as normal, whereas $67 \%$ reported values of $\mathrm{BuChE}$ activity within the range in a non-exposed population to anticholinesterase compounds. The results revealed an important variability in the values for $\mathrm{AChE}$ and $\mathrm{BuChE}$ activities, which show the need for continuous training among analysts and the homogenization of methodologies.
\end{abstract}

\section{INTRODUCCIÓN}

México ocupa el segundo lugar en Latinoamérica en cantidad de plaguicidas utilizados y el cuarto en grado de exposición con $4.5 \mathrm{~kg} /$ persona (Palacios Nava et al. 2009). Entre los plaguicidas más utilizados se encuentran los organofosforados (POF) y carbamatos (CB), cuyos principales efectos en la salud son producidos por la inhibición de las colinesterasas (ChEs) (Fulton y Key 2001, Costa 2006, Gupta y Milatovic 2012). Estas enzimas hidrolizan ésteres de colina y se clasifican de acuerdo con su localización tisular, especificidad de sustrato y por su inhibición con diferentes sustancias (Tecles et al. 2000). En el organismo existen dos tipos: la acetilcolinesterasa (AChE), colinesterasa verdadera o colinesterasa específica de tipo E, y la butirilcolinesterasa $(\mathrm{BuChE})$, pseudocolinesterasa, colinesterasa plasmática o colinesterasa de tipo $\mathrm{S}$ (Jeyaratnam y Maroni 1994).

La AChE (EC 3.1.1.7) se localiza principalmente en músculo, eritrocitos y sistema nervioso. Es la enzima responsable de la hidrólisis del neurotransmisor acetilcolina $(\mathrm{ACh})$ en colina y ácido acético en la sinapsis nerviosa (Massoulié et al. 1993, Schetinger et al. 2000). La inhibición de la AChE está vinculada directamente con el mecanismo de toxicidad de los POF y CB que bloquean la acción de esta enzima (Reigart y Roberts 1999). Los POF son capaces de fosforilar el sitio activo de la AChE; la proteína fosforilada ya no puede hidrolizar al neurotransmisor ACh y esto resulta en acumulación de la acetilcolina endógena y sobreestimulación de los receptores colinérgicos responsables de los signos y síntomas típicos (muscarínicos y nicotínicos) que ocurren después de la intoxicación aguda (Maroni et al. 2000, Bajgar 2004). Asimismo, se ha observado una fuerte asociación entre la exposición a plaguicidas y la disminución de la actividad $\mathrm{AChE}$ en poblaciones expuestas (Hernández et al. 2004, 2005, Rendón-von Osten et al. 2004, Gamlin et al. 2007, Remor et al. 2009).

$\mathrm{La} \mathrm{BuChE} \mathrm{(EC} \mathrm{3.1.1.8)} \mathrm{se} \mathrm{encuentra} \mathrm{en} \mathrm{diferentes}$ tejidos como intestino, hígado, riñón, corazón, pulmón y suero. Aunque su función fisiológica aún no está bien definida (Dave et al. 2000, Eddleston et al. 2008, Bodur y Layer 2011, Scacchi et al. 2011) se ha reportado que puede hidrolizar un número elevado de ésteres hidrofóbicos e hidrofílicos, de manera que podría actuar como un posible supresor endógeno de compuestos anticolinérgicos (Chuiko 2000). La inhibición de la actividad BuChE no está necesariamente asociada con los síntomas anticolinérgicos, ya que se ha observado una fuerte disminución de la actividad BuChE en ausencia de cualquier efecto sobre la AChE (Soltaninejad et al. 2007). Sin embargo, se ha propuesto que la BuChE podría ser un indicador de exposición más sensible que la $\mathrm{AChE}$ a ciertos POF (Ranjbar et al. 2002, Hernández et al. 2006, Rastogi et al. 2008, Jintana et al. 2009, Araoud et al. 2011, Bernal-Hernández et al. 2014). Asimismo, se ha descrito que la inhibición de la BuChE se relaciona con la intensidad y duración de la exposición, así como con el tipo de plaguicida (Araoud et al. 2011).

La determinación de la actividad de las colinesterasas es ampliamente utilizada en estudios de monitoreo biológico para evaluar la exposición y los efectos de compuestos anticolinesterásicos (Hernández et al. 2005, Safi et al. 2005, Souza et al. 2005, Ng et al. 2009, Stefanidou et al. 2009, 
Simoniello et al. 2010, Ueyama et al. 2010, BernalHernández et al. 2014). No obstante, se ha observado una amplia variabilidad en los valores considerados como normales para estas actividades enzimáticas, lo que puede deberse a diversos factores. Uno de los que más puede influir en la variabilidad de los datos es la falta de parámetros preanalíticos y analíticos estandarizados, ya que existen modificaciones al método convencional, y por otro lado hay laboratorios que preparan sus propios reactivos, mientras que otros emplean kits comerciales. Asimismo, la presencia de polimorfismos en estas enzimas puede afectar la función o expresión de la enzima (Hasin et al. 2005, Souza et al. 2005, Lockridge 2015). Estas modificaciones constituyen fuentes de variación, cuyo control es importante para mejorar la calidad y precisión de los resultados, así como para permitir una comparación entre las poblaciones estudiadas. En este sentido, el objetivo de este estudio fue conocer, con una metodología consensuada, los intervalos de las actividades AChE y BuChE en individuos no expuestos ocupacionalmente a compuestos anticolinesterásicos de diferentes estados del país.

\section{MATERIALES Y MÉTODOS}

Se realizó un ejercicio piloto para conocer las diferencias técnicas en la medición de la actividad $\mathrm{AChE}$ y BuChE entre laboratorios ubicados en diferentes universidades y centros de investigación en México, que miden la actividad de colinesterasas como biomarcador de exposición y efecto a plaguicidas anticolinesterásicos. En esta actividad participaron nueve laboratorios incluidos en la Red Temática de Toxicología de Plaguicidas, los cuales proceden de la Universidad Autónoma de Guerrero, el Centro de Investigación en Alimentación y Desarrollo, la Universidad Autónoma de Yucatán, Universidad Autónoma Metropolitana-Iztapalapa, Universidad Veracruzana, Universidad Autónoma de Tlaxcala y Universidad Autónoma de Nayarit.

Para la homogeneización de la metodología entre laboratorios se realizó la siguiente estrategia de trabajo:Se solicitó la metodología empleada en cada laboratorio participante. Mediante un cuestionario se obtuvo información acerca de reactivos empleados para evaluar las actividades AChE y BuChE, el sustrato e indicador utilizado, en qué matriz miden la actividad (sangre total, suero, plasma), la longitud de onda que se utiliza para medir la actividad, tiempos de incubación e intervalos de medición de la muestra, así como una descripción detallada de la toma de muestra, almacenamiento de la misma y forma de reportar la actividad. Lo anterior permitió conocer los factores preanalíticos y analíticos que pudieran influenciar la actividad y que son diferentes en cada laboratorio participante en el ejercicio.

Una vez recolectada la información, se hizo un análisis de las metodologías aplicadas y se unificaron criterios técnicos para la propuesta de una metodología que fue aplicada por todos los laboratorios interesados. Establecida la propuesta metodológica, se realizó un ejercicio entre todos los participantes para precisar el análisis de colinesterasas, mismo que se llevó a cabo en el Laboratorio de Biomembranas de la Universidad Autónoma Metropolitana-Iztapalapa.

Con la metodología consensuada, cada laboratorio analizó al menos 10 muestras de personas no expuestas ocupacionalmente a plaguicidas. Se generó una base de datos con los resultados y fueron analizados. Se incluyeron en el estudio, personas del sexo masculino, mayores de 18 años que no estuvieran ocupacionalmente expuestos a plaguicidas.

\section{Determinación de la actividad AChE Reactivos}

Amortiguador de fosfatos $\left(\mathrm{Na}_{2} \mathrm{HPO}_{4}-\mathrm{KH}_{2} \mathrm{PO}_{4}\right.$, $0.1 \mathrm{M}, \mathrm{pH}$ 7.4, J.T Baker), DNTB (10 mM, SigmaAldrich), etopropazina (6 mM, Sigma-Aldrich), tritón X-100 (0.03\%, Sigma-Aldrich) y yoduro de acetiltiocolina (28.3 mM, Sigma-Aldrich).

\section{Recolección y procesamiento de la muestra}

La medición de la actividad de AChE se realizó de acuerdo con la metodología establecida por Ellman et al. (1961), a partir de una muestra de sangre completa recolectada en tubos con EDTA como anticoagulante. Para realizar la lisis de los eritrocitos se preparó $1 \mathrm{~mL}$ de una dilución 1:100 de la muestra de sangre con el detergente no iónico tritón X-100.

\section{Procedimiento}

Se realizó una mezcla de reacción con los siguientes reactivos: $0.5 \mathrm{~mL}$ de la dilución $(1: 100)$ con $1 \mathrm{~mL}$ de amortiguador de fosfatos $(0.1 \mathrm{M}, \mathrm{pH} 7.4), 0.05$ $\mathrm{mL}$ de DTNB $(10 \mathrm{mM})$ y $0.005 \mathrm{~mL}$ de etopropazina $(6 \mathrm{mM})$. La mezcla se incubó a $37^{\circ} \mathrm{C}$ durante $10 \mathrm{~min}$, posteriormente se adicionaron $0.025 \mathrm{~mL}$ de yoduro de acetiltiocolina $(28.3 \mathrm{mM})$, se mezclaron y de manera inmediata se monitoreó el cambio de absorbancia a $436 \mathrm{~nm}$ cada minuto, durante $3 \mathrm{~min}$. Las mediciones se realizaron por triplicado. La actividad AChE fue corregida por el contenido de hemoglobina y se expresó como $\mathrm{U} / \mathrm{g}$ de $\mathrm{Hb}$. 


\section{Determinación de hemoglobina (Hb) Reactivos}

Reactivo de Drabkin (ferricianuro de potasio y cianuro de potasio, Hycel ${ }^{\circledR}$ )

\section{Procedimiento}

La concentración de $\mathrm{Hb}$ se determinó a partir de $10 \mu \mathrm{L}$ de sangre total que se mezclaron con $2.5 \mathrm{~mL}$ de reactivo de Drabkin. Posteriormente, la mezcla se incubó 10 min en oscuridad a temperatura ambiente y se midió su absorbancia a $540 \mathrm{~nm}$ frente a un blanco de reactivo de Drabkin.

\section{Determinación de la actividad BuChE Reactivos}

Amortiguador de fosfatos $\left(\mathrm{Na}_{2} \mathrm{HPO}_{4}-\mathrm{KH}_{2} \mathrm{PO}_{4}\right.$, 0.1 M, pH 7.4, J.T Baker), DTNB (10 mM, SigmaAldrich), yoduro de S-butiriltiocolina $(63.2 \mathrm{mM}$, Sigma-Aldrich).

\section{Recolección y procesamiento de muestra}

La medición de la actividad BuChE se realizó de acuerdo con la metodología propuesta por Ellman et al. (1961) a partir de una muestra de suero. Para obtener el suero, las muestras de sangre total se centrifugaron a $3500 \mathrm{rpm}$ durante $10 \mathrm{~min}$, se realizaron alícuotas y se almacenaron $\mathrm{a}-20^{\circ} \mathrm{C}$ hasta su análisis.

\section{Procedimiento}

Para medir la actividad $\mathrm{BuChE}$ se realizó una mezcla de $3 \mathrm{~mL}$ de amortiguador de fosfatos $(0.1 \mathrm{M}$ a pH 7.4), $0.100 \mathrm{~mL}$ de DTNB (10 mM) y $0.010 \mathrm{~mL}$ de suero. Se mezclaron cuidadosamente, se incubó a $37^{\circ} \mathrm{C}$ durante $10 \mathrm{~min}$ y posteriormente, se agregaron $0.050 \mathrm{~mL}$ de yoduro de S-butiriltiocolina $(63.2$ $\mathrm{mM}$ ). El cambio de absorbancia fue monitoreado a $405 \mathrm{~nm}$ durante $4 \mathrm{~min}$, cada minuto. La medición de la actividad enzimática se realizó por triplicado y se reportó en U/L.

\section{RESULTADOS Y DISCUSIÓN}

Los procedimientos analíticos para medir la actividad de las colinesterasas son muy diversos. El más empleado es el método colorimétrico de Ellman et al. (1961) que se basa en la detección de la tasa de desaparición del sustrato de la enzima o de aparición del producto de la reacción. Este método es aplicado en muestras de sangre, plasma, suero y tejidos de diversas especies. En este estudio se utilizaron muestras de sangre total y suero de individuos no expuestos ocupacionalmente a compuestos anticolinesterásicos. Los resultados de las mediciones fueron emitidos por cada laboratorio participante y analizados en conjunto. El cuadro I muestra la información obtenida a través del cuestionario sobre la metodología empleada en cada laboratorio para la determinación de colinesterasas. Con este ejercicio se identificó la variabilidad que existe en los factores preanalíticos y analíticos entre laboratorios. Todos los participantes en el ejercicio refirieron medir la actividad de colinesterasas a través del método de Ellman. Sin embargo, se identificaron diferencias en la concentración de sustrato y cromóforo utilizado, así como en la forma de reportar los resultados, lo que dificulta la comparación de los mismos entre poblaciones con características similares.

Los primeros métodos de medición de la actividad de colinesterasas fueron revisados por Witter en 1963, quien identificó que entre los errores implicados en la determinación de la actividad de estas enzimas se incluyen la posible contaminación de la muestra durante la recolección, la falta de centrifugación adecuada, así como el manejo y almacenamiento inadecuado de la muestra. Sin embargo, mencionó que eran necesarios más estudios para conocer el efecto del manejo preanalítico sobre la actividad de estas enzimas, particularmente sobre la actividad AChE (Witter 1963). Asimismo, un grupo de científicos convocados por la Agencia de Protección Ambiental estadounidense (EPA, por sus siglas en inglés) reconoció que no había un procedimiento operativo estándar para la determinación de la actividad de colinesterasas e implementaron un ejercicio entre laboratorios de investigación y clínicos, que permitió comparar las metodologías, estableciendo la reproducibilidad del método de Ellman (Wilson et al. 1996).

Utilizando la metodología propuesta por el grupo de trabajo se obtuvieron los siguientes resultados: la actividad promedio para la AChE fue de $23.6 \mathrm{U} / \mathrm{g}$ de $\mathrm{Hb}$ y de 12,247 U/L para la BuChE, valores que se encuentran dentro de los rangos considerados como normales (AChE:17-45 U/g de $\mathrm{Hb}, \mathrm{BuChE}(3,700-$ $13,200 \mathrm{U} / \mathrm{L}$ ). En este sentido, se observó que el $22 \%$ de los participantes reportaron valores de actividad AChE dentro del rango considerado como normal para una población no expuesta a compuestos anticolinesterásicos. Respecto a la actividad BuChE, el $67 \%$ de los participantes reportaron valores dentro del rango considerado como normal. En la figura 1 se muestra la dispersión de los valores reportados por cada laboratorio. Estudios previos han encontrado una alta variabilidad entre laboratorios, con frecuencia inaceptable, en ensayos basados en el método de Ellman (Mineau y Peakall 1987, Fleming et al. 1992, Wilson et al. 1996). En el presente estudio, 


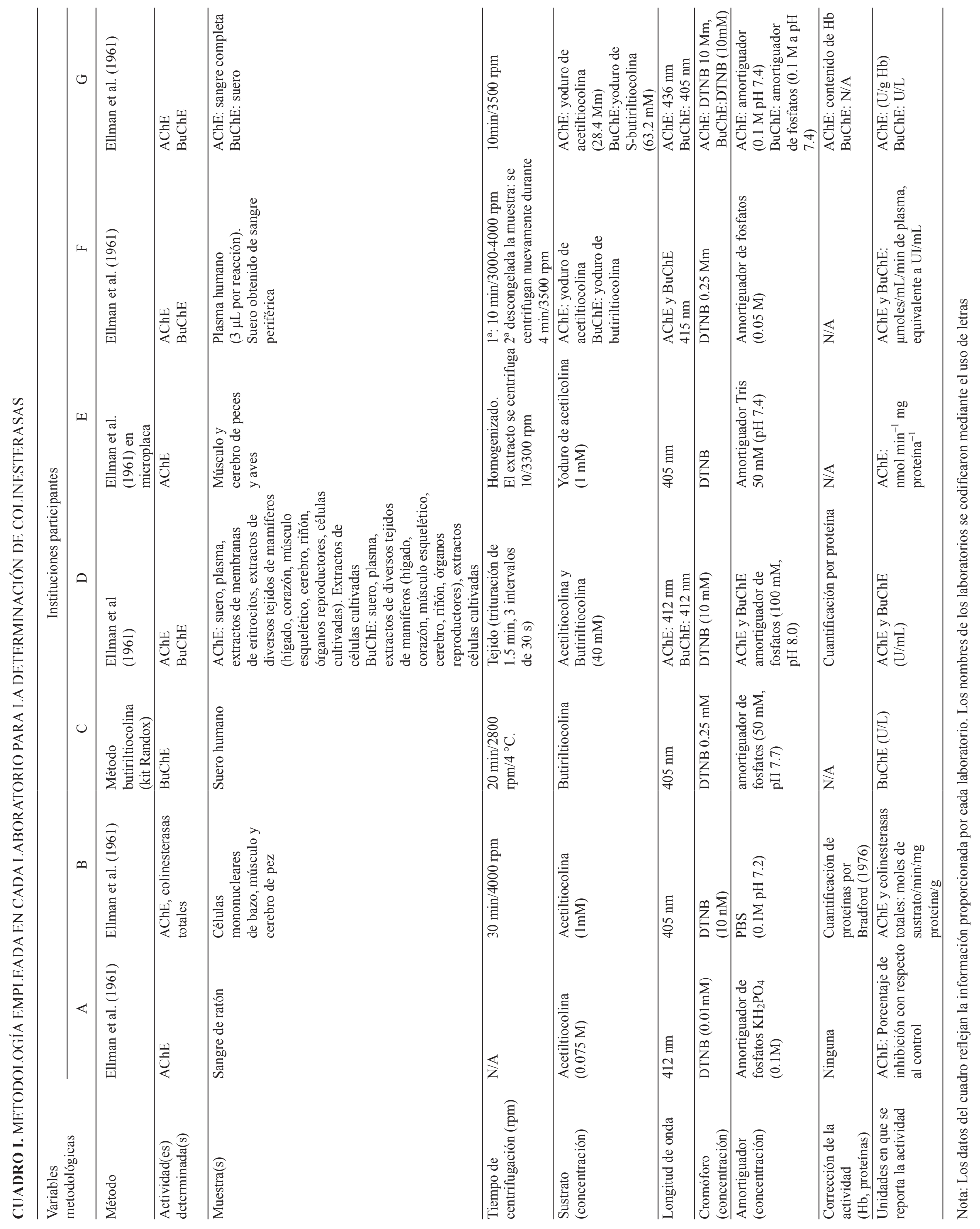



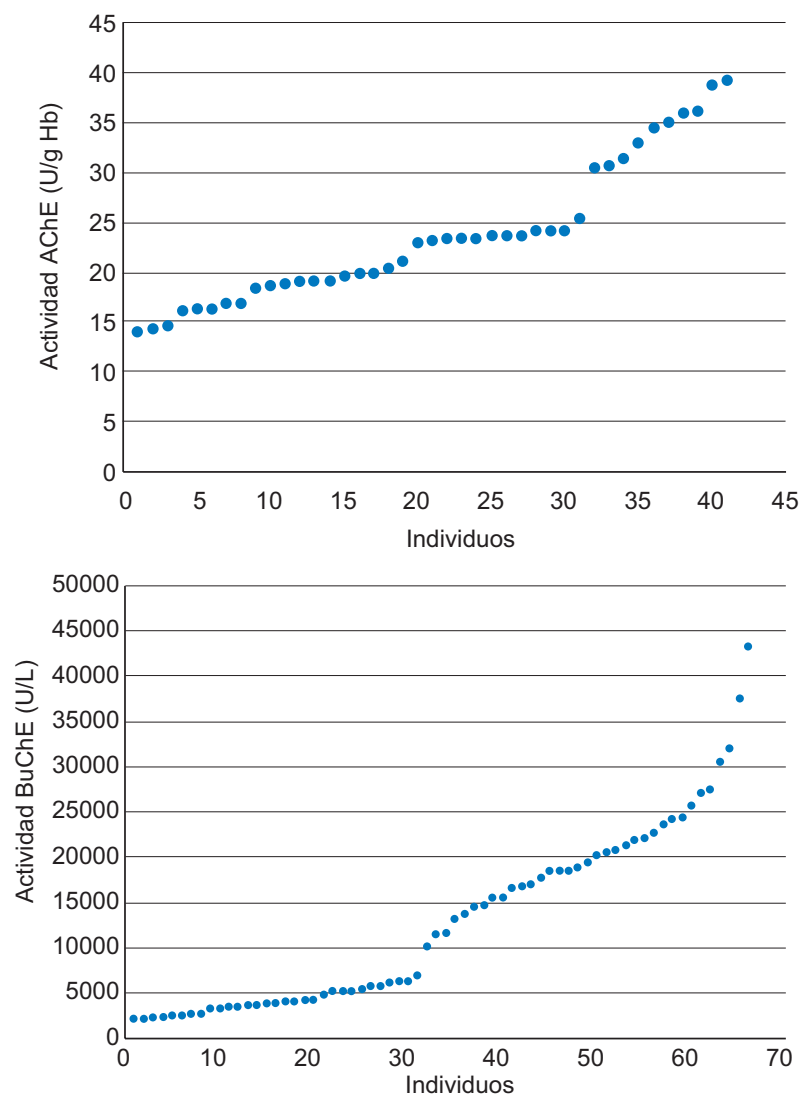

Fig. 1. Variabilidad de los resultados reportados por cada laboratorio

se observó una amplia dispersión de los datos para ambas colinesterasas aun cuando se estableció la metodología a seguir. Lo anterior pudiera deberse, por un lado, a factores preanalíticos y analíticos, y por otro a factores propios del individuo, como la presencia de polimorfismos en los genes $B u C h E y$ $A C h E$. Estas alteraciones genéticas pueden afectar la función o expresión de la enzima, cuya actividad se ve alterada (Nogueira et al. 1992, LoewensteinLichtenstein et al. 1995, Yen et al. 2003, Sánchez et al. 2005). Lo anterior evidencia la necesidad de establecer un procedimiento operativo estándar como el que se propone en este ejercicio, que permita la homogeneización de las metodologías utilizadas en cada laboratorio y facilite la comparación de resultados.

\section{CONCLUSIÓN}

Existen diversos factores preanalíticos y analíticos que influyen en la medición de la actividad de colinesterasas (AChE y BuChE). Por tanto, es necesario realizar más esfuerzos para homogeneizar la metodología establecida, con el fin de controlar su influencia y optimizar tanto la precisión como la exactitud de los resultados. Asimismo, la propuesta metodológica constituye una alternativa precisa y barata para la determinación de colinesterasas. Sin embargo, en este ejercicio se observó una amplia variabilidad en los resultados reportados, lo que muestra la necesidad de capacitación continua entre analistas y la homogenización de metodologías, con el fin de obtener una mejor comparación de estos biomarcadores bioquímicos.

\section{AGRADECIMIENTOS}

Los autores agradecen a la Red Temática de Toxicología de Plaguicidas (CONACyT$262284 / 280045 / 294303$ ) y a los participantes de este estudio.

\section{REFERENCIAS}

Araoud M., Neffeti F., Douki W., Ben-Hfaiedh H., Akrout M., Najjar M.F. y Kenani A. (2011). Factors influencing plasma butyrylcholinesterase activity in agricultural workers. Ann. Biol. Clin. 69, 159-166.

DOI:10.1684/abc.2011.0531

Bajgar J. (2004). Organophosphates / nerve agent poisoning: mechanism of action, diagnosis, prophylaxis, and treatment. Adv. Clin. Chem. 38, 151-216.

DOI: 10.1016/S0065-2423(04)38006-6

Bernal-Hernández Y.Y., Medina-Díaz I.M., BarrónVivanco B.S., Robledo-Marenco M.L., Girón-Pérez M.I., Pérez-Herrera N.E. y Rojas-García A.E. (2014). Paraoxonase 1 and its relationship with pesticide biomarkers in indigenous Mexican farmworkers. J. Occup. Environ. Med. 56, 281-290.

DOI: 10.1097/01.jom.0000438381.25597.8

Bodur E. y Layer P.G. (2011). Counter-regulation of cholinesterases: differential activation of PKC and ERK signaling in retinal cells through BChE knockdown. Biochimie 93, 469-476.

DOI: $10.1016 /$ j.biochi.2010.10.020

Chuiko G.M. (2000). Comparative study of acetylcholinesterase and butyrylcholinesterase in brain and serum of several freshwater fish: specific activities and in vitro inhibition by DDVP, an organophosphorus pesticide. Comp. Biochem. Phys. C. 127, 233-242. DOI: $10.1016 / \mathrm{S} 0742-8413(00) 00150-\mathrm{X}$

Costa L.G. (2006). Current issues in organophosphate toxicology. Clin. Chim. Acta. 366, 1-13. DOI: $10.1016 /$ j.cca.2005.10.008 
Dave K.R., Syal A.R. y Katyare S.S. (2000). Tissue cholinesterases. A comparative study of their kinetic properties. Z. Naturforsch. C. 55, 100-108.

DOI: 10.1515/znc-2000-1-219

Eddleston M., Eyer P., Worek F., Sheriff M.R. y Buckley N.A. (2008). Predicting outcome using butyrylcholinesterase activity in organophosphorus pesticide self-poisoning. Q. J. Med. 101, 467-474.

DOI: 10.1093/qjmed/hen026

Ellman G., Courtney K.D., Andres V. Jr. y Feather-Stone R.M. (1961). A new and rapid colorimetric determination of acetylcholinesterase activity. Biochem. Pharmacol. 7, 88-95. DOI: 10.1016/0006-2952(61)90145-9

Fleming, R.A., Holmes, S.B., y Busby, D.G. (1992). An inter laboratory compar ison of data on brain cholinesterase activity in forest songbirds exposed to aerial application of Zectranâ. Arch. Environ. Contam. Toxicol. 22, 228-237

Fulton M.H. y Key P.B. (2001). Acetylcholinesterase inhibition in estuarine fish and invertebrates as an indicator of organophosphorus insecticide exposure and effects. Environm. Toxicol. Chem. 20, 37-45.

DOI: $10.1002 /$ etc. 5620200104

Gamlin J., Romo P.D. y Hesketh T. (2007). Exposure of young children working on Mexican tobacco plantations to organophosphorous and carbamic pesticides, indicated by cholinesterase depression. Child. Care. Health. Dev. 33, 246-248.

DOI: $10.1111 / j .1365-2214.2006 .00702 . x$

Gupta R.C. y Milatovic D. (2012). Toxicity of organophosphates and carbamates. En: Mammalian toxicology of insecticides, vol. 104. (Gupta R.C., Ed). Royal Society of Chemistry, Cambridge, UK, pp. 573-585.

Hasin Y., AvidanN., Bercovich D., Korczyn A.D., Silman I., Beckmann J.S. y Sussman J. (2005). Analysis of genetic polymorphisms in acetylcholinesterase as reflected in different populations. Curr. Alzheimer Res. 2, 207-218

Hernández A.F., Gómez M.A., Pérez V., García-Lario J.V., Pena G., Gil F. y Pla A. (2006). Influence of exposure to pesticides on serum components and enzyme activities of cytotoxicity among intensive agriculture farmers. Environ. Res. 102, 70-76.

DOI: 10.1016/j.envres.2006.03.002

Hernández A.F., López O., Rodrigo L., Gil F., Pena G., Serrano J.L. y Pla A. (2005). Changes in erythrocyte enzymes in humans long-term exposed to pesticides: influence of several markers of individual susceptibility. Toxicol. Lett. 159, 13-21.

DOI: 10.1016/j.toxlet.2005.04.008

Hernández A.F., Gómez M.A., Pena G., Gil F., Rodrigo L., Villanueva E. y Pla A. (2004). Effect of long-term exposure to pesticides on plasma esterases from plastic greenhouse workers. J. Toxicol. Environ. Health A. 67, 1095-1108. DOI: $10.1080 / 15287390490452371$

Jintana S., Sming K., Krongtong Y. y Thanyachai S. (2009). Cholinesterase activity, pesticide exposure and health impact in a population exposed to organophosphates. Int. Arch. Occ. Env. Health 82, 833-842. DOI: $10.1007 / \mathrm{s} 00420-009-0422-9$

Jeyaratnam J. y Maroni M. (1994) Organophosphorous compounds. Toxicology 994(91), 15-27.

Loewenstein-Lichtenstein Y., Schwarz M., Glick D., Norgaard-Pedersen B., Zakut H. y Soreq H. (1995). Genetic predisposition to adverse consequences of anticholinesterases in 'atypical' BCHE carriers, Nat. Med. 1(10), 1082e1085.

Lockridge O. (2015). Review of human butyrylcholinesterase structure, function, genetic variants, history of use in the clinic, and potential therapeutic uses. Pharmacol. Therapeut. 148, 34-46.

Maroni M., Colosio C., Ferioli A. y Fait A. (2000). Introduction. Toxicology 143, 5-118. DOI: $10.1016 / \mathrm{S} 0300-483 X(99) 00152-3$

Massoulié J., Pezzementi, L., Bon, S., Krejci, E. y Vallette, F.M. (1993). Molecular and cellular biology of cholinesterases. Prog. Neurobiol. 41, 31-91.

DOI: 10.1016/0301-0082(93)90040-Y

Mineau P. y Peakall D.B. (1987). An evaluation of avian impact assessment techniques following broad-scale forest insecticide sprays. Environ. Toxicol. Chem. 6, 781-791.

Ng V., Koh D., Wee A. y Chia S.E. (2009). Salivary acetylcholinesterase as a biomarker for organophosphate exposure. Occup. Med. 59, 120-122.

DOI: $10.1093 / \mathrm{occmed} / \mathrm{kqn} 164$

Nogueira C.P., Bartels C.F., McGuire M.C., Adkins S., Lubrano T., Rubinstein H.M., Lightstone H., Van der Spek A. F., Lockridge O y La Du B.N. (1992) Identification of two different point mutations associated with the fluoride-resistant phenotype for human butyrylcholinesterase. Am. J. Hum. Genet. 51(4), 821-828.

Palacios Nava M.E, García de la Torre G.S, Paz Román M.P. (2009). Determinación de niveles basales de colinesterasa en jornaleros agrícolas. Rev. Fac. Med. UNAM 52(2), 63-68.

Ranjbar A.K.R.A.M., Pasalar P. y Abdollahi M. (2002). Induction of oxidative stress and acetylcholinesterase inhibition in organophosphorous pesticide manufacturing workers. Hum. Exp. Toxicol. 21, 179-182.

DOI: $10.1191 / 0960327102 \mathrm{ht} 238 \mathrm{oa}$

Rastogi S.K., Singh V.K., Kesavachandran C., Siddiqui M.K.J., Mathur N. y Bharti R.S. (2008). Monitoring of plasma butyrylcholinesterase activity and hematological parameters in pesticide sprayers. Indian J. Occup. Environ. Med. 12, 29.

DOI: $10.4103 / 0019-5278.40813$ 
Reigart J.R. y Roberts J.R. (1999). Recognition and management of pesticide poisonings. 5a ed. U.S Environmental Protection Agency, Washington DC, 239 pp.

Remor A.P., Totti C.C., Moreira D.A., Dutra G.P., Heuser V.D. y Boeira J.M. (2009). Occupational exposure of farm workers to pesticides: biochemical parameters and evaluation of genotoxicity. Environ. Int. 35, 273278. DOI: 10.1016/j.envint.2008.06.011

Rendón-Von Osten. J., Epomex C., Tinoco-Ojanguren R., Soares A.M. y Guilhermino L. (2004). Effect of pesticide exposure on acetylcholinesterase activity in subsistence farmers from Campeche, México. Arch. Environ. Occup. Health 59, 428-435.

DOI: 0.3200/AEOH.59.8.418-425

Safi J.M., Mourad T.A. y Yassin M.M. (2005). Hematological biomarkers in farm workers exposed to organophosphorus pesticides in the Gaza Strip. Arch. Environ. Occup. Health 60, 235-241. DOI: 10.3200/AEOH.60.5.235-241

Scacchi R., Ruggeri M. y Corbo R.M. (2011). Variation of the butyrylcholinesterase (BChE) and acetylcholinesterase (AChE) genes in coronary artery disease. Clin. Chim. Acta 412, 1341-1344.

DOI: $10.1016 /$ j.cca.2011.03.033

Schetinger M.R., Porto N.M., Moretto M.B., Morsch V.M., da Rocha J.T., Vieira V. y Zanatta N. (2000). New benzodiazepines alter acetylcholinesterase and ATPDase activities. Neurochem. Res. 25, 949-955. DOI: 10.1023/A:1007500424392

Sánchez L.H., Medina O., Gómez G., González C.L., Flórez-Vargas O. (2015). Laboratory genetic-based reference values for cholinesterase activity in a Colombian population: A step forward in personalized diagnostics. Biomédica 35(Supl.2), 20-29.

DOI: $10.7705 /$ biomedica.v 35 i0.2422

Simoniello M.F., Kleinsorge E.C., Scagnetti J.A., Mastandrea C., Grigolato R.A., Paonessa A.M. y Carballo
M.A. (2010). Biomarkers of cellular reaction to pesticide exposure in a rural population. Biomarkers 15, 52-60. DOI: 10.3109/13547500903276378

Soltaninejad K., Shadnia S., Afkhami-Taghipour M., Saljooghi R., Mohammadirad A. y Abdollahi M. (2007). Blood $\beta$-glucuronidase as a suitable biomarker at acute exposure of severe organophosphorus poisoning in human. Hum. Exp. Toxicol. 26, 963-966.

DOI: $10.1177 / 0960327107085349$

Souza M.S., Magnarelli G.G., Rovedatti M.G., Cruz S.S. y D'Angelo A.P.D. (2005). Prenatal exposure to pesticides: analysis of human placental acetylcholinesterase, glutathione S-transferase and catalase as biomarkers of effect. Biomarkers 10, 376-389.

DOI: $10.1080 / 13547500500272614$

Stefanidou M., Athanaselis S. y Spiliopoulou H. (2009). Butyrylcholinesterase: biomarker for exposure to organophosphorus insecticides. Int. Med. J. 39, 57-60. DOI: 10.1111/j.1445-5994.2008.01779.x

Tecles F., Subiela S.M., Bernal L.J. y Cerón J.J. (2000). Use of whole blood for spectrophotometric determination of cholinesterase activity in dogs. Vet. J. 160, 242-249. DOI: 10.1053/tvj1.2000.0499

Ueyama J., Satoh T., Kondo T., Takagi K., Shibata E., Goto M. y Kamijima M. (2010). $\beta$-Glucuronidase activity is a sensitive biomarker to assess low-level organophosphorus insecticide exposure. Toxicol. Lett. 193, 115-119. DOI: 10.1016/j.toxlet.2009.12.009

Wilson B. W. (1996). Factors in standardizing automated cholinesterase assays. J. Tox. Environ. Health 48(2), 187-196, DOI: 10.1080/009841096161429

Witter R.F. (1963). Measurement of blood cholinesterase. Arch. Environ. Health 6, 537-563.

Yen T., Nightingale B.N., Burns J.C., Sullivan D.R, Stewart P.M. (2003). Butyrylcholinesterase (BCHE) genotyping for post-succinylcholine apnea in an Australian population. Clin. Chem. 49(8) 1297-1308. 